\title{
Magnetic Resonance Imaging Evaluation of Spinal Infections
}

\author{
Vulchi Ranjith Kumar ${ }^{\circledR 1}$, Rama Krishna Rao Baru ${ }^{\odot 2}$ \\ ${ }^{1}$ Post Graduate, Department of Radio-Diagnosis, Narayana Medical College \& Hospital, Nellore, Andhra Pradesh, India, ${ }^{2}$ Professor, Department of Radio-Diagnosis, \\ Narayana Medical College \& Hospital, Nellore, Andhra Pradesh, India.
}

\section{Abstract}

Background: The advantage of Magnetic resonance imaging include multiplanar capabilities and soft-tissue contrast resolution, which is superior to that of CT. Magnetic resonance (MR) imaging is a powerful diagnostic tool that can be used to help evaluate spinal infection and to help distinguish between an infection and other clinical conditions. Aim of the current study is to evaluate various spectrum and types of spinal infections, and discussing the role of MRI in diagnosing them and their characterization. Subjects \& Methods: This Hospital-based prospective study consists 30 patients with clinically suspected spinal infections and chronic non-resolving low backache referred to the department of Radiodiagnosis in a period of 2 years. Investigations include Complete blood count, ESR, sputum analysis for acid-fast bacilli and MRI of the spine. Results: 20 cases involved the lumbar spine, of which 12 were tubercular, seven were pyogenic, and one case was actinomycosis. In total 21 tubercular cases, 12 cases involved lumbar spine (57\%), 8 cases affects the thoracic spine (38\%), and 1 case involves the cervical spine ( $\mathrm{P}$ $=0.562)$. the incidence of spondylodiscitis is common overall in the lumbar spine. $23.8 \%$ of tubercular and $12.5 \%$ of pyogenic cases involved more than two vertebrae. T1 hypointensity is seen in 18 cases of tuberculosis $(85 \%), 8$ cases of pyogenic (75\%), and 1 case of actinomycosis $(100 \%)(\mathrm{P}=0.801) .4$ cases showed preservation of disc height, among which three are tubercular $(75 \%)$, and 1 was actinomycosis $(25 \%)$. $85 \%$ of tubercular and $100 \%$ of pyogenic cases showed disc narrowing. $81 \%$ of tubercular and $100 \%$ of pyogenic cases showed disc hyperintensity. Nine cases of tuberculosis $(42.9 \%)$ and 3 cases of pyogenic $(37.5 \%)$ showed epidural abscess. 26 cases showed para vertebral extension of which 18 were tubercular $(69.2 \%)$, 7 were pyogenic $(26.9 \%)$ and 1 was actinomycosis $(3.8 \%) .94 \%$ of tubercular and $42 \%$ of pyogenic abscesses showed a well-defined para spinal signal in cases of paraspinal extension. 15 of the 18(83\%) tuberculosis, 3 of the 7 (42\%)cases of pyogenic, and 1 case of actinomycosis showed subligamentous spread along more than three vertebrae. Heterogenous enhancement was noted in 12 of the $15(80 \%)$ tubercular cases, 1 of the $3(33 \%)$ pyogenic cases, and $1(100 \%)$ actinomycosis case. $71 \%$ tubercular cases and 2 of 8 (25\%) cases showed predominant anterior $2 / 3^{r d}$ involvement. Grade III or more $(>50 \%)$ vertebral destruction was seen in 16 tubercular $(76 \%)$ and 2 pyogenic cases (25\%). Six cases showed skip lesions of which 5were tubercular and 1 was pyogenic. 5 of the 21 (23.8\%) tubercular and 1 of the $8(12.5 \%)$ pyogenic cases showed skip lesions. Conclusion: Awareness of atypical MR imaging at early infectious spondylitis is important to avoid diagnostic delay and unnecessary other diagnostic procedures. Several non-infectious conditions may simulate the spinal infections. Hence It is helpful to be aware of these diseases and their MR imaging features. With these points in mind, MR imaging can be very beneficial to patients with spinal infection.

Keywords: Spinal Infection, Magnetic Resonance Imaging, Tubercular, Pyogenic.

Corresponding Author: Rama Krishna Rao Baru, Professor, Department of Radio-Diagnosis, Narayana Medical College \& Hospital, Nellore, Andhra Pradesh, India.

E-mail: drkdurga60@gmail.com

Received: 25 August 2020

\section{Introduction}

Spinal infections have increased since the 1990s due to aging populations, higher numbers of immunocompromised hosts longer life expectancy in patients with chronic illnesses, intravenous drug users, and increased use of instrumentations for spinal surgery and epidural catheters for pain treatment. ${ }^{[1]}$

Easy availability of MRI has contributed to the increasing number of patients with a definitive diagnosis of spinal infections. ${ }^{[2]}$

Staphylococcus aureus is the most frequent organism found in cases of pyogenic spinal infections and followed by streptococcal species. ${ }^{[3]}$

Staphylococcus epidermidis has been recognized as a cause of spinal diseases associated with implants such as prosthetic materials and pacemakers. ${ }^{[4]}$

Methicillin-resistant Staphylococcus aureus(MRSA) has been reported to account for $45 \%$ to $61 \%$ of $S$. aureus cases of spinal 
infections. ${ }^{[5]}$

Another study demonstrated that methicillin-resistant S. aureus or S.epidermidis was isolated approximately 2.5 times more frequently in patients over age $80 .{ }^{[6]}$

Infections of the spine are the conditions that cause high mortality and morbidity when they are not diagnosed and treated accurately. Neurological deficits and permanent deformities of the spine are the sequelae of delayed diagnosis and even can lead to death. ${ }^{[7]}$ They may involve various structures, including bone, disc, epidural, subdural, subarachnoid spaces, and also affects the spinal cord.

Classification of spinal infections is mainly based on etiology into bacterial, fungal, viral, and parasitic infections. ${ }^{[8]}$

Clinical suspicion of spinal disease is made in patients with fever, raised ESR, and backache. The confirmation of the diagnosis is by imaging modalities such as radiographs, CT, MRI, radionuclide bone imaging Magnetic resonance imaging is the imaging modality of choice for the diagnosis of clinically suspected cases of spinal infections. ${ }^{[9]}$

MRI contrast study is the modality of choice for the anatomical localization and early diagnosis of spinal infections. ${ }^{[10]}$

Multiplanar capabilities and soft tissue contrast resolution are the advantages of MRI. ${ }^{11}$ It is also useful for delineating the extent of spread of disease into the paravertebral and epidural spaces.

Aim of the current study is to evaluate various spectrum and types of spinal infections, and discussing the role of MRI in diagnosing them and their characterization.

\section{Subjects and Methods}

The primary source of data was patients attending the department of Radiodiagnosis, Narayana Medical College, Nellore. This Hospital-based prospective study consists 30 patients with clinically suspected spinal infections.

\section{Methodology}

All patients referred to the department of Radiodiagnosis, aged between 20-80 years irrespective of sex, with the following criteria in a period of 2 years are taken for the study.

\section{Inclusion Criteria}

The study includes

- Patients with suspected clinical spinal infections.

- Patients with chronic non-resolving low backache.

\section{Exclusion Criteria}

The study will exclude
- The patient's having a history of claustrophobia.

- Patient having a history of metallic implants insertion, cardiac pacemakers, and metallic foreign body in situ.

- Patients with known primary malignancies causing low backache.

\section{Technique}

All patients presenting with symptoms of spinal infection referred from emergency, outpatient, or inpatient department will be studied on 3.0 Tesla GE MRI scanner (Discovery MR750w). The following sequences will be performed, sagittal T1 and T2weighted images, axial T2 weighted images, and STIR images and Gadolinium T1 weighted images are acquired whenever necessary with approximate acquisition time of 15 to 20 minutes.

The sample size of thirty patients was examined generally and neurologically.

\section{Investigations done included}

Complete blood count (CBC), ESR, three consecutive sputum analysis for acid-fast bacilli and MRI of the spine.

\section{Results}

Thirty cases have been included in the study, of which 21 were tubercular, eight were pyogenic, and 1 was actinomycotic spondylodiscitis.

The study was done on 30 patients, of which 21 were tubercular, eight were pyogenic, and one was actinomycosis.

\section{Sex distribution}

In our study group of 30 patients, 15 were males, and 15 were females.

Among 15 males, nine were of tubercular etiology, and five were of pyogenic spondylodiscitis. And one case proved to be actinomycotic spondylodiscitis. Among 15 females, 12 were of tubercular, and three were pyogenic.

Of all the 21 tubercular cases, 12 were females, and 9 were males.

Of all the eight pyogenic cases, 5 were males, and 3 were females.

In our study, the incidence of spondylodiscitis is equal in males and females. Tuberculosis is seen commonly in females than males. Whereas pyogenic spondylodiscitis is seen often in males (Chi-Square value $=1.929, \mathrm{P}$ Value $=0.381$ (Not Sig.).

\section{Site of involvement}

20 cases involved the lumbar spine, of which 12 were tubercular, seven were pyogenic, and one case was actinomycosis.

Nine cases involved the thoracic spine, of which eight were tubercular, and one was pyogenic. 
One case involved the cervical spine, which was diagnosed as tuberculosis.

Of all the 21 tubercular cases, 12 cases involved lumbar spine (57\%), 8 cases affects the thoracic spine (38\%), and 1 case involves the cervical spine (Chi-Square $=2.977, \mathrm{p}=0.562$ ( Not Sig.).

Of all the eight pyogenic cases, $7(87.5 \%)$ cases involved the lumbar spine, 1 case (12.5\%) involved the thoracic spine, and one case of actinomycosis involved the lumbar spine.

In our study, the incidence of spondylodiscitis is common overall in the lumbar spine.

The most common involvement of the thoracic spine is with tuberculosis $(88.9 \%)$.

\section{Number of vertebrae involved}

Involvement of 2 vertebrae is noted in 23 cases, more than two vertebrae in 6 cases, and one vertebra in one case.

Of the 6 cases that involved more than two vertebrae, five were tubercular (83.3\%), and one was pyogenic (16.7\%).

Of the 23 cases that involved two vertebrae, 15 were tubercular $(65.2 \%)$, seven were pyogenic $(30.4 \%)$ and 1 was actinomycosis $(4.3 \%)$.

Among 21 tubercular cases 15 cases involved only two vertebrae (71.4\%), 5 cases involved more than two vertebrae (23.8\%), 1 case involved only one vertebra (4.8\%).

7 among 8 pyogenic cases involved only two vertebrae $(87.5 \%), 1$ case involved more than two vertebrae $(12.5 \%)$. One case of actinomycosis involved two vertebrae $(100 \%)$ (Chi-Square value $=0.813, \mathrm{P}$ Value $=0.937$ (Not Sig.).

The number of vertebrae involved in both tubercular and pyogenic is predominantly two vertebrae $(71.4 \%$ and $87.5 \%$ respectively.)

\section{Signal intensity}

T1 hypointensity is seen in 18 cases of tuberculosis (85\%), 8 cases of pyogenic (75\%), and 1 case of actinomycosis $(100 \%)$ (Chi-Square value $=0.443, \mathrm{P}$ Value $=0.801$ (Not Sig.).

Reduced disc height: 4 cases showed preservation of disc height, among which three are tubercular (75\%), and 1 was actinomycosis $(25 \%)($ Chi-Square value $=7.747, \mathrm{P}$ Value $=$ 0.021 (Sig.).

Among 26 cases that showed the decreased height of 18 were tubercular (69.2\%), eight were pyogenic (30.8\%).

Eighteen cases of tuberculosis $(85.7 \%)$ showed reduced disc height, and all the 8 cases $(100 \%)$ showed decreased height in pyogenic.

Preservation of disc height is mostly seen in tubercular and actinomycosis cases.
T2 hyperintensity in seen in 20 cases of tuberculosis (95\%), 8 cases of pyogenic $(100 \%)$, and 1 case of actinomycosis $(100 \%)$.

Predominant signal intensity is $\mathrm{T} 1$ hypo and $\mathrm{T} 2$ hyper in both tubercular and pyogenic, whereas $\mathrm{T} 2$ hyperintensity is more in pyogenic than the tubercular.

In our study, $95 \%$ of tubercular and $100 \%$ of pyogenic cases showed T2 hyperintensity, and $100 \%$ of tubercular and pyogenic cases showed $\mathrm{T} 1$ hypointensity.

In our study, $85 \%$ of tubercular and $100 \%$ of pyogenic cases showed disc narrowing.

\section{Altered disc intensity}

5 cases do not show altered disc intensity, of which four were tubercular, and one case was actinomycosis.

Among 25 cases that showed altered signal intensity, 17 cases were tubercular, 8 cases were pyogenic (Chi-Square value $=$ 6.686, $\mathrm{P}$ Value $=0.035$ (Sig.).

Seventeen cases of tuberculosis (81\%), and all cases of pyogenic showed altered signal intensity.

Preservation of disc with no altered signal intensity is mostly seen in tuberculosis and actinomycosis.

In our study, $81 \%$ of tubercular and $100 \%$ of pyogenic cases showed disc hyperintensity.

Epidural abscess: 12 cases showed epidural abscess, among which $9(75 \%)$ were tubercular, three were pyogenic $(25 \%)$ (Chi-Square value $=0.759, \mathrm{P}=0.684$ (Not Sig.).

Nine cases of tuberculosis (42.9\%) and 3 cases of pyogenic (37.5\%) showed epidural abscess.

8 of the $9(88 \%)$ tuberculosis and 1 of the 3 (33\%) pyogenic abscess showed smooth abscess wall.

Paravertebral extension: 26 cases showed para vertebral extension of which 18 were tubercular $(69.2 \%), 7$ were pyogenic $(26.9 \%)$ and 1 was actinomycosis $(3.8 \%)$ (ChiSquare value $=0.175, \mathrm{P}$ Value $=0.916($ Not Sig. $)$.

18 cases of tuberculosis (85.7\%), 7 cases of pyogenic (87.5) and 1 case of actinomycosis (100\%) showed paravertebral extension.

\section{Paravertebral signal:}

17 of the $18(94 \%)$ cases of tuberculosis, 3 of the $7(42 \%)$ cases of pyogenic, and 1 case of actinomycosis with paravertebral extension showed well-defined para spinal signal.

\section{Sub ligamentous spread:}

15 of the $18(83 \%)$ tuberculosis, 3 of the $7(42 \%)$ cases of pyogenic, and 1 case of actinomycosis showed subligamentous spread along more than three vertebrae.

Endplate changes were noted in all cases of tuberculosis, pyogenic, and actinomycosis cases. 
Kyphosis noted in 7 cases, of which all were tubercular origin.

7 of the 21 tuberculosis cases showed kyphosis (33 $3 \%$ )

Pyogenic and actinomycosis do not show kyphosis in any of the cases.

The mean age for tuberculosis is $49.6 \mathrm{yrs}$; pyogenic is 51.13 , and actinomycosis is $47 \mathrm{yrs}$.

Loss of cortical definition: 17 of the 21(80\%) tuberculosis cases, 3 of the $8(37.5 \%)$ pyogenic cases, and $1(100 \%)$ actinomycosis case showed loss of cortical definition.

\section{Vertebral involvement:}

In our study, 15 of 21 (71\%) tubercular cases and 2 of $8(25 \%)$ cases showed predominant anterior $2 / 3^{r d}$ involvement.

The actinomycosis case showed entire vertebral body involvement with no anterior or posterior predilection.

\section{Post-contrast:}

Post-contrast study was done in 19 cases of which 15 were tubercular, three were pyogenic, and 1 was actinomycosis.

\section{Vertebral destruction :}

Grade III or more ( $>50 \%$ ) vertebral destruction was seen in 16 tubercular (76\%) and 2 pyogenic cases $(25 \%)$.

\section{Skip lesions:}

Six cases showed skip lesions of which 5were tubercular and 1 was pyogenic.

5 of the 21 (23 8\% tubercular and 1 of the 8 (12 5\% pyogenic cases showed skip lesions.

\section{Discussion}

Several imaging modalities are available for the evaluation of spinal infections. These include plain films of the spine, computed tomography, radionuclide scintigraphy, myelography, CT following myelography, and magnetic resonance imaging (MRI). MRI is generally considered the best imaging modality to evaluate spinal infections.

The study was done on 30 patients, of which 21 were tubercular, eight were pyogenic, and one was actinomycosis. Among 15 males, nine were of tubercular etiology, and five were of pyogenic spondylodiscitis and one case proved to be actinomycotic spondylodiscitis. Among 15 females, 12 were of tubercular, and three were pyogenic. In a study by Ritu dhawan et al (2015) on 50 cases, the sex distribution of infectious spondylodiscitis is $1.9: 1 .^{[11]}$

In our study, $38 \%$ of tubercular and 12.5 of pyogenic cases involved the thoracic spine, whereas $57 \%$ of tubercular and $87.5 \%$ of pyogenic cases involved the lumbar spine. Whereas in a study by Yuko Harada et al. (2008), 60\% of tubercular and $7.7 \% \%$ of pyogenic cases involved the thoracic spine. In our study, $23.8 \%$ of tubercular and $12.5 \%$ of pyogenic cases involved more than two vertebrae. Whereas In a study by Ritu dhawan et al. (2015) on 50 cases, $25 \%$ of tubercular and $22 \%$ of pyogenic cases involved more than two vertebrae. In a study by Ritu Dhawan et al. (2015), 43.8\% of tubercular cases and $33.5 \%$ of pyogenic cases showed involvement of 2 vertebrae. ${ }^{[11]}$

In our study, 95\% of tubercular and $100 \%$ of pyogenic cases showed T2 hyperintensity, and $100 \%$ of tubercular and pyogenic cases showed T1 hypointensity. Ritu dhawan et al. (2015) on 50 cases, $81 \%$ of tubercular and $80 \%$ of pyogenic cases showed T1 hypointensity. $40 \%$ of tubercular and $60 \%$ of pyogenic cases showed T2 hyperintensity. ${ }^{[11]}$

In our study, $85 \%$ of tubercular and $100 \%$ of pyogenic cases showed disc narrowing. In a study by Ritu dhawan et al. (2015) on 50 cases, $55 \%$ of tubercular and $81 \%$ of pyogenic cases showed disc narrowing.

In our study, $81 \%$ of tubercular and $100 \%$ of pyogenic cases showed disc hyperintensity. In a study by Na young Jung et al. (2004), $80 \%$ of tubercular and $90 \%$ of pyogenic cases showed disc hyperintensity. ${ }^{[12]}$

In our study, $88 \%$ of tubercular and $33 \%$ of pyogenic abscesses showed a smooth wall. In a study by Yuko Harada et al. (2008), $90 \%$ of tubercular and $7.7 \%$ of pyogenic abscesses showed a smooth wall. ${ }^{[13]}$

In our study, $94 \%$ of tubercular and $42 \%$ of pyogenic abscesses showed a well-defined para spinal signal in cases of paraspinal extension. In a study by Ritudhawan et al. (2015) on 50 cases, $80 \%$ of tubercular and $40 \%$ of pyogenic abscesses showed a well-defined para spinal signal in cases of paraspinal extension.

In our study, $83 \%$ of tubercular and $42 \%$ of pyogenic cases with paravertebral extension showed sub ligamentous spread more than three vertebrae. In a review by Yuko Harada et al. (2008), $50 \%$ of tubercular and $53 \%$ of pyogenic cases with paravertebral extension showed sub ligamentous spread more than three vertebrae.

In our study, the mean age for tuberculosis is $49.6 \mathrm{yrs}$; pyogenic is 51.13 , and actinomycosis is $47 \mathrm{yrs}$.

In our study, $80 \%$ of tubercular and $37.5 \%$ of pyogenic cases showed a loss of cortical definition. In a study by Ritudhawan et al. (2015) on 50 cases, $75 \%$ of tubercular and $20 \%$ of pyogenic cases showed loss of cortical definition.

In our study, 15 of $21(71 \%)$ tubercular cases and 2 of 8 $(25 \%)$ cases showed predominant anterior $2 / 3^{r d}$ involvement. Upon Post-contrast study was done in 19 cases, heterogenous enhancement was noted in 12 of the $15(80 \%)$ tubercular cases, 1 of the $3(33 \%)$ pyogenic cases, and $1(100 \%)$ actinomycosis case. 

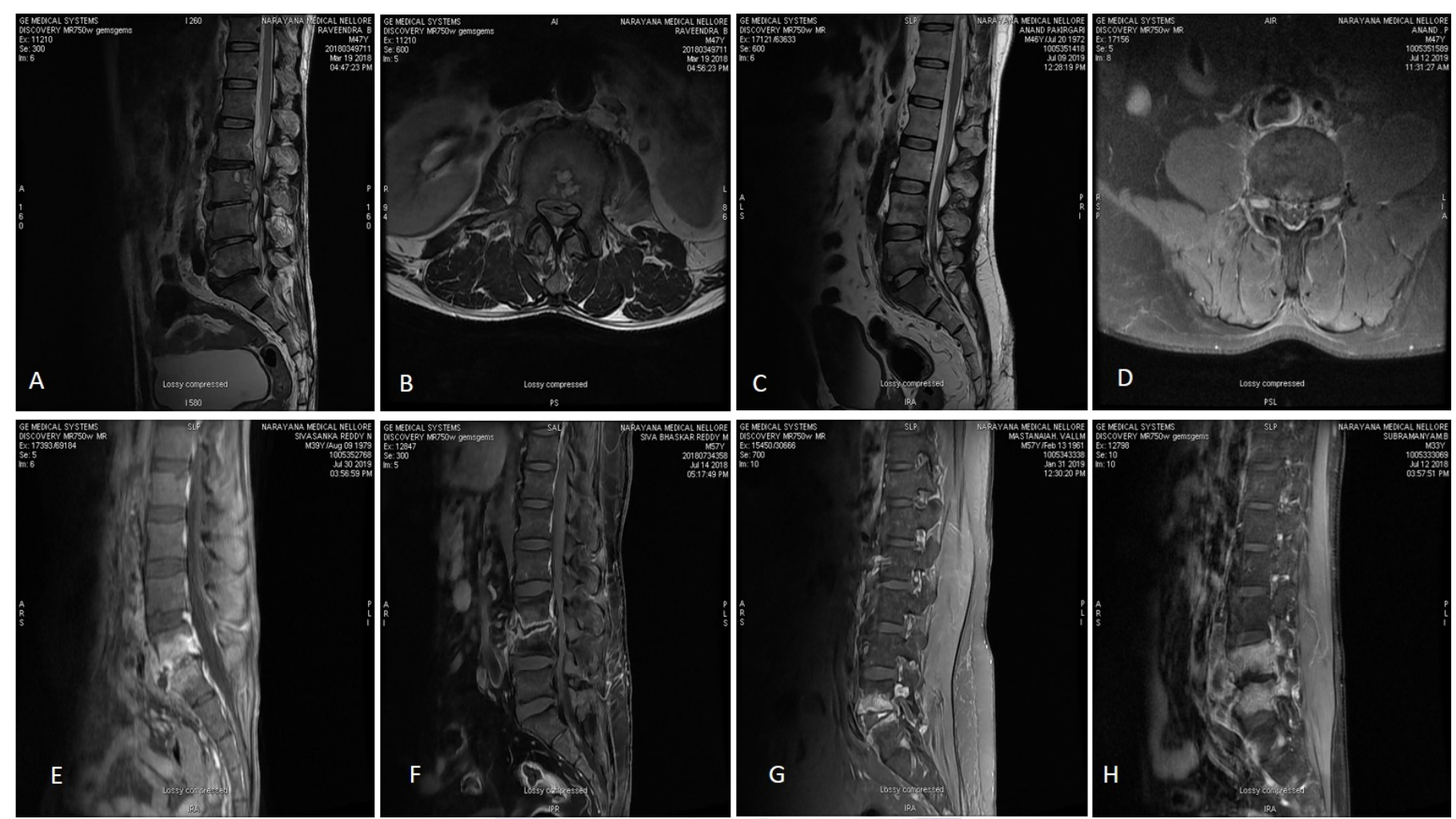

Figure 1: A. Prototype cases. Sag T2 image showing mild hyperintensity at the corresponding level. B. Axial T2 image showing paravertebral soft tissue component. C. Pyogenic spondylodiscitis. Sag T1 image showing hypointensity at L5S1 level with sparing of the intervertebral disc and prevertebral component. Sag T2 image showing hypointensity at this level. D. Axial post-contrast. (patient 2). E. Fungal osteomyelitis. (actinomycosis). Sag post-contrast imageshowing heterogenous enhancement with well-defined peripherally enhancing prevertebral and left paraspinal abscess at this level (Patient 3). F. Pyogenic Spondylodiscitis with Epidural abscesses. Sag post-contrast images showing heterogenous enhancement predominantly at the endplates with paravertebral extension and presacral abscesses (patient 4). G. Pyogenic spondylodiscitis. Sag image showing heterogeneous enhancement of the endplates and minimal paravertebral extension (Patient 5). H. Sag post-contrast images showing heterogeneous enhancement of the vertebrae with enhancing prevertebral collection.

Grade III or more $(>50 \%)$ vertebral destruction was seen in 16 tubercular (76\%)and 2 pyogenic cases $(25 \%)$. In a study conducted by Chang et al. 2006, 82\% of tubercular and 30 $\%$ of pyogenic cases showed grade III or more vertebral destruction. ${ }^{[14]}$

In our study Six cases showed skip lesions of which 5 were tubercular and 1 was pyogenic.

In our study, 5 of the $21(23.8 \%)$ tubercular and 1 of the $8(12.5 \%)$ pyogenic cases showed skip lesions. In a study conducted by Ritudhawan et al. (2015), $25 \%$ of tubercular and $11.1 \%$ of pyogenic cases showed skip lesions

Contrast-enhanced sequences help distinguish between tuberculous spondylitis, and other granulomatous spinal infections, the presence of a thick rim of enhancement around paraspinal and intraosseous abscesses is reported to be diagnostic of tuberculous spondylitis. The involvement of the paraspinal soft tissues and the subligamentous spread of infection are also important features of tuberculous spondylitis. ${ }^{[15]}$

Dagirmanjian et al., 1999, stated that the main MRI imaging features suggestive of tuberculous spondylitis are: ${ }^{[16]}$

1. The infection usually involves the ventral trabecular bone marrow adjacent to the intervertebral disc. In this study, the infection typically affects the ventral superior and inferior parts of the vertebral bodies. Posterior element involvement (pedicle, laminae, and spinous process) did not occur in any of our cases.

2. The infection spreads via the anterior longitudinal ligament to adjacent vertebral bodies. In our study, the 


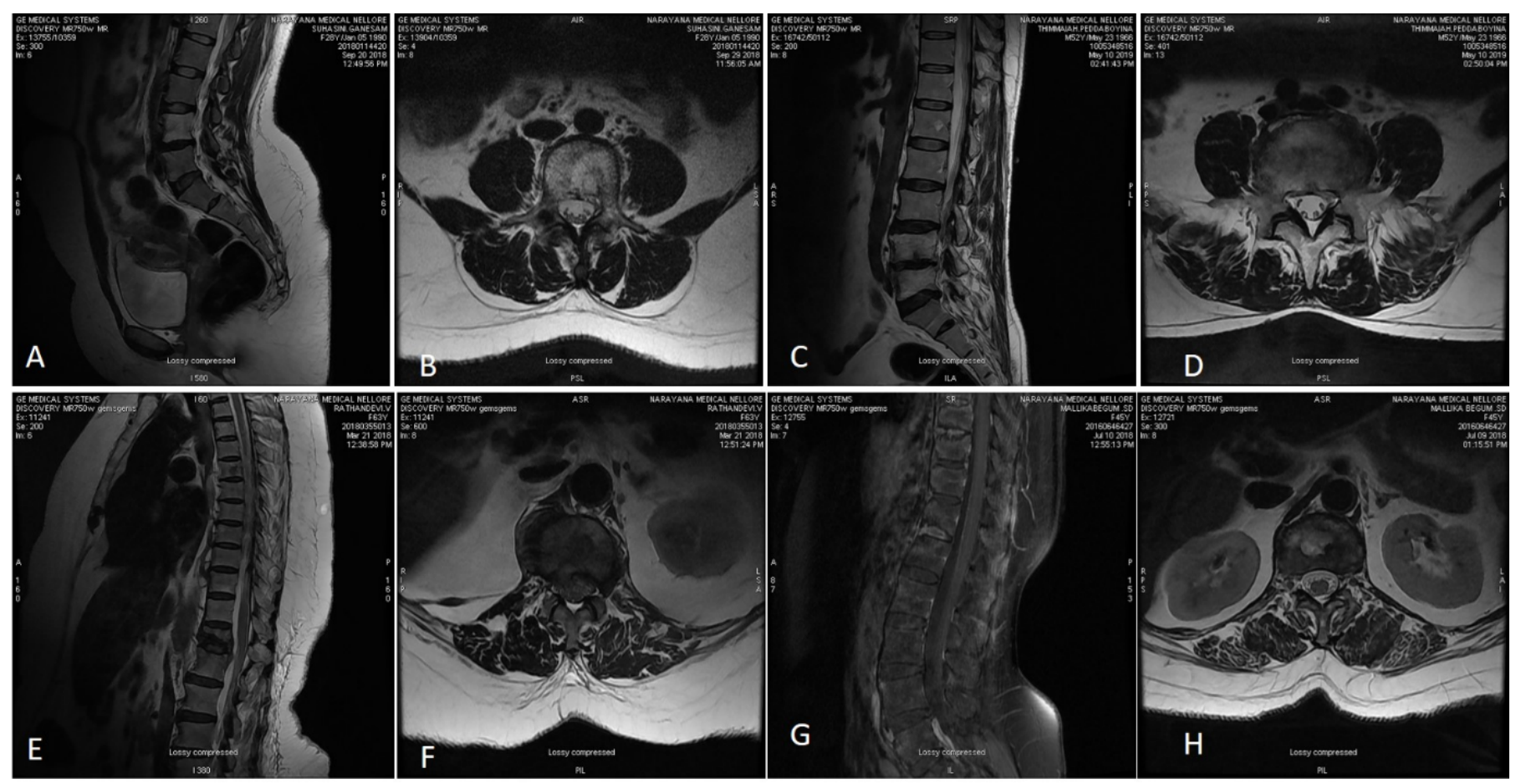

Figure 2: A. TB spondylitis with sparing of the disc. Sag T2 images showing hyperintensity involving the L4 vertebra with sparing of the disc (patient 7). B. Axial T2 image showing hyperintensity of the vertebra(patient 7). C. Potts spine with the fatty replacement of vertebrae. T2 hyperintensity involving the L4-L5 level with narrowing of the disc space (patient 8).D. Axial T2 image showing mild paravertebral extension. E. Pyogenic spondylodiscitis involving the Dorso-lumbar spine with paravertebral collections. Sag T2 image showing hyperintensity at this level.F. Axial T2 image showing pre and right paravertebral soft tissue component. G. Tubercular spondylodiscitis with enhancing paravertebral collection. Sag and H. Axial post-contrast images showing mild heterogenous enhancement at this level with no reduction in disc space(patient 10).

extension of infection beneath the anterior longitudinal ligament occurs (subligamentous extension).

3. Disc involvement is relatively limited and late compared with the amount of vertebral body involvement.

4. Large paraspinal soft tissue mass, which is usually out of proportion to the amount of vertebral body involvement.

Factors favoring tuberculous spondylodiscitis over pyogenic spondylodiscitis includes involvement of more than two continuous vertebrae, Thoracic spine involvement, however, in our study, most common site is the lumbar spine, Late involvement of the disc with few cases showing disc sparing, Well defined para spinal signal, Smooth abscess wall, Sub ligamentous extension of more than three vertebral levels, Loss of cortical definition, Predominant involvement of anterior part of the vertebrae, Heterogenous contrast enhancement of the granulation tissue, Moderate to severe vertebral destruction, and Skip lesions and kyphosis.
As regarding pyogenic spondylitis, Smith, and Blaser (1991) reported that MR imaging of pyogenic spondylitis typically shows low signal intensity on T1-weighted images with loss of definition of the endplate and the adjacent vertebral bodies. This is caused by the replacement of the normal fatty marrow by the inflammatory tissue and the development of necrotic areas. ${ }^{[17]}$

Also, following intravenous gadolinium, disc enhancement patterns include homogeneous enhancement of most of the disk, patchy nonconfluent areas of disc enhancement, or thick or thin areas of peripheral disc enhancement. ${ }^{[18]}$

Significant MRI findings that indicate pyogenic infection includes decreased signal of the marrow of two adjacent vertebrae with loss of definition of the intervening end plates on T1-WI; the contiguous involvement of the disc space that is the hallmark of pyogenic infection. On T1WI, this is seen as the loss of the ability to discern between the disc and the vertebral body, while on T2WI, it is seen as abnormal high 
signal intensity within the interspace; epidural abscess that typically extends two to three vertebral segments in length.

However, Sharif et al. stated that the differentiation of tuberculous spondylitis from pyogenic spondylitis could sometimes be difficult. ${ }^{[19]}$

Clinical data in such cases are invaluable in suggesting the likely pathology, with a more insidious onset of signs and symptoms favoring tuberculous infections.

Factors favoring pyogenic spondylodiscitis over tubercular includes Predominant lumbar spine involvement; Involvement of 2 or less than two vertebrae; Ill-defined para spinal signal intensity; Early involvement of the disc; Thick and irregular abscess wall; Less vertebral destruction (less than grade III); Homogenous contrast enhancement post contrast; and Involvement of the entire vertebral body.

\section{Conclusion}

The routine sagittal and axial sequences employed are usually T1and T2-weighted fast spin-echo sequences. Also, gadolinium $\mathrm{T} 1$ images are useful in differentiating inflammation from abscess, necrosis and bone sequestra as a result of infection and also in helping to localize and detect subtle areas of inflammation within the vertebral bodies, meninges, spinal cord, and nerve roots. Rim enhancement around intraosseous or extraosseous abscesses following gadolinium administration, heterogeneous contrast enhancement, severe vertebral collapse, and gibbus deformity.

Pyogenic spondylitis is characterized by Decreased signal of the marrow of two adjacent vertebrae with loss of definition of the intervening end plates on T1-WI, the contiguous and early involvement of the disc space, epidural abscess that typically extends two to three vertebral segments in length , ill-defined para spinal signal, thick and irregular abscess wall, homogenous contrast enhancement.

Actinomycotic spondylodiscitis is rare and is similar to imaging features of tubercular spondylodiscitis with relative preservation of disc space.

\section{References}

1. Maiuri F, Gallicchio B, Manto A, Briganti F. Spondylodiscitis: clinical and magnetic resonance diagnosis. Spine. 1997;22(15):1741-1747. Available from: https://doi.org/10. 1097/00007632-199708010-00012.

2. Stefanovski N, Voris LPV. Pyogenic vertebral osteomyelitis: report of a series of 23 patients. Contemp Orthop. 1995;31(3):159-164.

3. Chew FS, Kline MJ. Diagnostic Yield of CT-guided Percutaneous Aspiration Procedures in Suspected Spontaneous Infectious Diskitis. Radiology. 2001;218(1):211-214. Available from: https://dx.doi.org/10.1148/radiology.218.1.r01ja06211.

4. Cheung WY, Luk KDK. Pyogenic spondylitis. International Orthopaedics. 2012;36:397-404. Available from: https://dx. doi.org/10.1007/s00264-011-1384-6.

5. Acharya J, Gibbs WN. Imaging spinal infection. Radiol Infect Dis. 2016;3:84-91. Available from: https://dx.doi.org/10.1016/ j.jrid.2016.03.001.

6. Reyes-Prieto A, Barquera B, Juárez O. Origin and Evolution of the Sodium -Pumping NADH: Ubiquinone Oxidoreductase. PLoS ONE. 2014;9:96696. Available from: https://dx.doi.org/ 10.1371/journal.pone.0096696.

7. Ruiz A, Post MJ, Sklar EM, Holz A. MR imaging of the infections of the cervical spine. Magn Reson Imaging Clin N Am. 2000;8(3):561-580.

8. Smith AS, Blaser SI. Infectious and inflammatory processes of the spine. Radiol Clin North Am. 1991;19:809-827.

9. The diagnosis of infections of the spine by modern imaging techniques. Orth Clin North Am. 1996;27(1):15-31.

10. Talbott JF, Shah VN, Uzelac A, Narvid J, Dumont RA, Chin $\mathrm{CT}$, et al. Imaging-Based Approach to Extradural Infections of the Spine. Semin Ultrasound CT MR. 2018;39(6):570-586. Available from: https://dx.doi.org/10.1053/j.sult.2018.09.003.

11. Galhotra R, Jain T, Sandhu P, Galhotra V. Utility of magnetic resonance imaging in the differential diagnosis of tubercular and pyogenic spondylodiscitis. J Nat Sci Biol Med. 2015;6(2):388. Available from: https://dx.doi.org/10.4103/ 0976-9668.160016.

12. Jung NY, Jee WH, Ha KY, Park CK, Byun JY. Discrimination of tuberculous spondylitis from pyogenic spondylitis on MRI. Am J Roentgenol. 2004;182(6):1405-1415. Available from: https://doi.org/10.2214/ajr.182.6.1821405.

13. Harada Y, Tokuda O, Matsunaga N. Magnetic resonance imaging characteristics of tuberculous spondylitis vs. pyogenic spondylitis. Clin Imaging. 2008;32:303-309. Available from: https://dx.doi.org/10.1016/j.clinimag.2007.03.015.

14. Chang MC, Wu HT, Lee CH, Liu CL, Chen TH. Tuberculous spondylitis and pyogenic spondylitis: comparative magnetic resonance imaging features. Spine. 2006;31(7):782-790. Available from: https://doi.org/10.1097/01.brs.0000206385. 11684.d5.

15. Shanely DJ. Tuberculosis of the spine: imaging features. Am J Roentgenol. 1995;164:659-664. Available from: https://doi. org/10.2214/ajr.164.3.7863889.

16. Dagirmanjian A, Schils J, McHenry M, Modic MT. MR imaging of vertebral osteomyelitis revisited. Am J Roentgenol. 1996;167(6):1539-1543. Available from: https://dx.doi.org/10. 2214/ajr.167.6.8956593.

17. Smith AS, Blaser SI. Infectious and inflammatory processes of the spine. Radiol Clin North Am. 1991;29:809-827.

18. Varma R, Lander P, Assaf A. Imaging of Pyogenic Infectious Spondylodiskitis. Radiol Clin North Am. 2001;39(2):203213. Available from: https://dx.doi.org/10.1016/s00338389(05)70273-6.

19. Sharif HS. Role of MR imaging in the management of spinal infections. Am J Roentgenol. 1992;158(6):1333-1345. Available from: https://dx.doi.org/10.2214/ajr.158.6.1590137. 
Copyright: (C) the author(s), 2020. It is an open-access article distributed under the terms of the Creative Commons Attribution License (CC BY 4.0), which permits authors to retain ownership of the copyright for their content, and allow anyone to download, reuse, reprint, modify, distribute and/or copy the content as long as the original authors and source are cited.

How to cite this article: Kumar VR, Baru RKR. Magnetic Resonance Imaging Evaluation of Spinal Infections. Asian J. Med. Radiol. Res. 2020;8(2):80-87.

DOI: dx.doi.org/10.47009/ajmrr.2020.8.2.13

Source of Support: Nil, Conflict of Interest: None declared. 